\title{
Implementación de la educación virtual en tiempos de COVID-19 en la Facultad de Música de la UANL
}

\author{
Implementation of virtual education in times of COVID-19 at the Faculty of \\ Music of the UANL
}

\begin{abstract}
Implementação da educação virtual em tempos de COVID-19 na Faculdade de Música da UANL
\end{abstract}

\author{
Beania Salcedo Moncada \\ Universidad Autónoma de Nuevo León, México \\ beania_salcedo@hotmail.com \\ https://orcid.org/0000-0002-5653-9625 \\ José María López Prado \\ Universidad Autónoma de Nuevo León, México \\ jmlopezprado@hotmail.com \\ http://orcid.org/0000-0001-9175-8914
}

\section{Resumen}

Esta investigación se hizo con la finalidad de contar un diagnóstico que describa las percepciones del alumnado y los profesores de la Facultad de Música de la Universidad Autónoma de Nuevo León (UANL) sobre la capacitación para el uso de los recursos digitales durante el confinamiento provocado por la covid-19. Para este trabajo de investigación cualitativa se utilizó el diseño de la investigación-acción. Los resultados arrojaron que la mayoría de los encuestados cuenta con una red segura de internet; que muchos estudiantes no tenían habilitado el correo institucional, necesario para la utilización de la plataforma Microsoft Teams, pero lograron solucionarlo con prontitud. Además, se manifestó la falta de capacitación en la utilización de las plataformas digitales. En cuanto a las experiencias adquiridas, se lograron implementar estrategias que probaron el grado de innovación y 


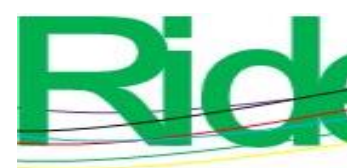

Revista Iberoamericana para la Investigación y el Desarrollo Educativo ISSN $2007-7467$

testaram o grau de inovação e criatividade dos professores para abordar conteúdos temáticos durante a formação em tempos de covid-19. Por fim, por meio da análise do discurso, observou-se que a educação a distância tem apresentado diferenças socioeconômicas e que ainda há a necessidade de se obter uma formação adequada no uso das tecnologias digitais que se ajustem à educação musical.

Palavras-chave: covid-19, diagnóstico acadêmico, educação musical, estratégia virtual. Fecha Recepción: Enero 2021 Fecha Aceptación: Julio 2021

\section{Introducción}

La educación en línea se ha implementado a partir de la emergencia sanitaria derivada de la covid-19, que tuvo sus inicios en la provincia de Hubei, China, a finales del 2019, y se expandió precipitadamente por el resto del mundo. "Evidentemente, la extensión geográfica y la velocidad a la que se dispersan las enfermedades infecciosas han crecido grandemente y esto no es más que el reflejo de la movilidad humana actual” (Torales y Munguía, 2020, párr. 7).

El 11 de marzo de 2020, profundamente preocupada por los alarmantes niveles de propagación y de gravedad que conlleva, la Organización Mundial de la Salud [OMS] (2020) calificó a la enfermedad por coronavirus del 2019 (covid-19) como pandémica, y recomendó el distanciamiento social como la principal medida de prevención.

El Gobierno de México, a través de la Secretaría de Educación Pública [SEP] (2020), publicó en el Diario Oficial de la Federación [DOF] (16 de marzo de 2020) el "Acuerdo número 02/03/20 por el que se suspenden las clases en las escuelas de educación preescolar, primaria, secundaria, normal y demás para la formación de maestros de educación básica del sistema educativo nacional, así como aquellas de los tipos medio superior y superior dependientes de la SEP".

La Universidad Autónoma de Nuevo León (UANL) emitió un comunicado el 14 de marzo de 2020 en el que se informó la decisión de suspender las clases presenciales a partir del 17 de marzo y hasta nuevo aviso, debido a los nuevos casos en la entidad y como medida de prevención y mitigación de la pandemia (Milenio Digital, 14 de marzo de 2020). Allí mismo, la UANL anunció que las actividades académicas continuarían en línea para dar cumplimiento a lo dispuesto en los diversos programas educativos que oferta. 


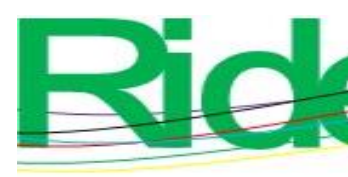

Revista Iberoamericana para la
Investigación y el Desarrollo Educativo
ISSN $2007-7467$

Sin embargo, antes de iniciar las clases en esta nueva modalidad, la Facultad de Música de la UANL realizó un diagnóstico a profesores y estudiantes para conocer el nivel de capacitación que tenía la comunidad académica en la utilización de las plataformas digitales propuestas a nivel institucional, los programas educativos en los que trabajaba cada profesor y la accesibilidad de internet de cada integrante de la Facultad. Una vez hecho el anterior diagnóstico, el 20 de abril se reanudaron las clases en línea a través de la plataforma Microsoft Teams para continuar con el semestre y se hizo un reajuste en el calendario académico con el objetivo de cumplir con el contenido total de los planes y programas de estudio programados para el ciclo escolar.

Cabe mencionar que la plataforma Microsoft Teams, que se propone para el uso en todos los niveles educativos de la UANL, forma parte de la estrategia digital de transformar y habilitar los procesos de enseñanza-aprendizaje presenciales a la modalidad en línea. Microsoft Teams es una plataforma unificada de comunicación y colaboración que permite realizar videollamadas, cuenta con almacenamiento de archivos e integra aplicaciones que fortalecen el quehacer educativo. Para acceder a esta plataforma, es necesario que cada estudiante o profesor tenga habilitada su cuenta de correo institucional.

"El aporte digital avalado por más de 20 años de experiencia, la dirección de educación digital, garantiza la excelencia de sus colaboradores como resultado de la implementación de nuestra amplia variedad de servicios" (UANL, 2020b, párr.2).

A través de tutoriales y sesiones en línea, se realizaron capacitaciones a estudiantes y profesores para la implementación de la plataforma Microsoft Teams. En efecto, la Facultad de Música de la UANL publicó el 21 de abril de 2020 un manual para docentes de Microsoft Teams. Se trata de un compilatorio de diferentes apartados explicativos para lograr un aprendizaje significativo que estimule la formación de estudiantes con un pensamiento crítico que les permita adaptarse a las nuevas necesidades y cambios sociales.

\section{Una nueva realidad en la educación}

La realidad social, económica y educativa durante esta pandemia será un punto de referencia para una nueva normalidad. Actualmente, las tecnologías conforman el andamiaje que permite seguir con los contenidos académicos en los centros educativos a nivel internacional. 

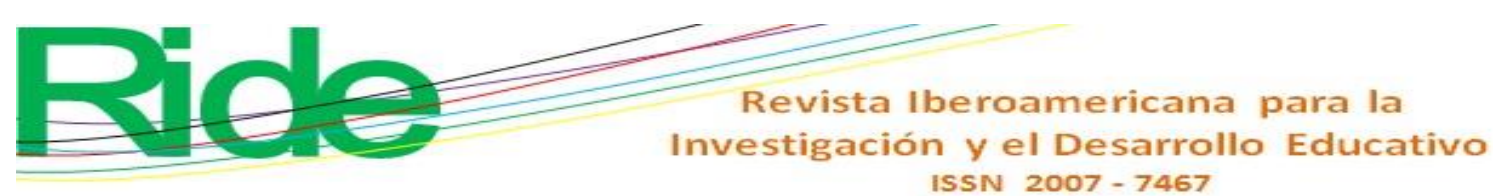

"Durante mucho tiempo, los programas de música y la educación musical en sí han permanecido inmunes a la investigación e innovación pedagógica. Hoy en día, esta situación está dando un importante giro" (Giráldez, 2010, p. 145).

Koutsoupidou (2014) opina que el entrenamiento musical puede ser examinado desde diferentes perspectivas, tomando en cuenta factores como la edad de las personas involucradas, el nivel de educación, la naturaleza del aprendizaje (formal, informal), campos específicos de instrucción musical y el modo de instrucción.

La Facultad de Música de la UANL ofrece la licenciatura en Música con acentuación en composición, instrumentista, educación musical, canto y dirección coral. La mayoría de las clases optativas son de manera individual, porque hacen referencia al aprendizaje del instrumento que cada estudiante escogió.

Las estrategias utilizadas para las clases de instrumento siempre han estado centradas en el aprendizaje autónomo, en donde el profesor funge como guía facilitador, permitiendo que el estudiante construya su propio conocimiento a partir de las bases técnico-musicales que le permitan desarrollar habilidades fisiológicas y cognitivas para el estudio de su instrumento.

Durante el confinamiento propiciado por la covid-19, las estrategias utilizadas se han ajustado a una nueva enseñanza-aprendizaje. El alumno se ha vuelto aún más autónomo y el maestro ha tenido que elaborar un andamiaje desde la distancia, utilizando plataformas y programas que se adapten a su materia y que permitan abarcar el total de los contenidos del programa.

En el caso puntual de las clases de instrumento de la Facultad de Música, que comprenden una parte primordial del mapa curricular de los estudiantes de los niveles técnico medio en Música, técnico superior universitario y licenciatura en Música, la plataforma propuesta por la UANL, Microsoft Teams, no ha resultado del todo práctica: el sonido se desplaza continuamente en las videollamadas, no se aprecia la postura corporal del estudiante y en ocasiones tampoco se puede ver con claridad la posición de las manos.

Para un estudiante de música es de capital importancia un desarrollo físico que le permita obtener las habilidades necesarias para tocar un instrumento y un desarrollo auditivo musical que le permita reconocer las formas de la literatura musical. Por este motivo, dependemos de la calidad de la videollamada para poder instruir al alumno en sus clases individuales de forma sincrónica. "El desarrollo de la audición es para los músicos lo que el desarrollo visual para los artistas plásticos" (Malbrán, 2007, p. 124). 


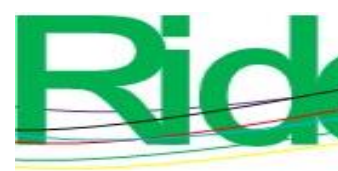

Revista Iberoamericana para la
Investigación y el Desarrollo Educativo
ISSN $2007-7467$

Los elementos de la música son el sonido, el ritmo, la intensidad y la altura. Los estudiantes deben desarrollar una técnica adecuada para el refinamiento muscular que les permita lograr la emisión del sonido de su instrumento. Es necesario mantener un constante entrenamiento auditivo con una visión crítica. "La escucha intencional tiene un objetivo particular o propósito, aprender algo o para usarla de alguna manera después de que la experiencia de escucha terminó" (Green, 2019, p. 40). Cuando el estudiante logra desarrollar la habilidad de escuchar analíticamente, puede implementar elementos didácticos en el trabajo de la emisión del sonido.

Durante el confinamiento es sustancial la optimización del tiempo para lograr cumplir con las cargas que cada materia comprende y con la práctica del instrumento, puesto que para adquirir las habilidades necesarias para el abordaje e interpretación de las obras propuestas en el programa de cada semestre se requiere de mucho tiempo y constancia. "Una enseñanza con menos presencia del profesor y menos localizada en el aula es toda una invitación a la autonomía de las personas, a su capacidad para organizar sus tiempos” (Feito, 2020, p. 162).

Se ha comprobado, a través de la experiencia, que, debido a la premura con que se implementó la educación a distancia, ha faltado preparación y capacitación por parte de los profesores para ofrecer los contenidos de forma virtual a los estudiantes. Evidentemente, la planeación de las clases y las estrategias utilizadas han tenido que cambiar debido a la nueva modalidad.

Algo no menos importante es que no todos los alumnos cuentan con las herramientas necesarias para continuar su formación a distancia, por ejemplo, la falta de internet en casa, los dispositivos, que muchas veces tienen que compartir con sus hermanos o con los padres que también están trabajando en línea. Aunado a estas circunstancias, también hay estudiantes de piano que no tienen instrumento en su casa y que hacían su práctica diaria en la Facultad de Música. "Pretender hoy que el sistema educativo realice adecuadamente su función a distancia es una fantasía que conduce a la frustración e incremente las desigualdades educativas" (Rogero, 2020, p. 175). 


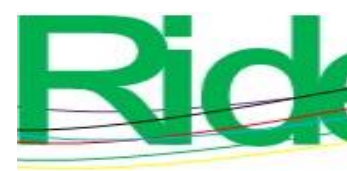

Revista Iberoamericana para la Investigación y el Desarrollo Educativo ISSN $2007-7467$

\section{Adaptación del sistema educativo durante COVID-19}

Cárdenas y Valdés (15 de abril de 2020) señalan que "la expectativa de un cambio acelerado de conductas de jóvenes y adultos para hacer frente al Covid-19, resalta la oportunidad que tendremos para reposicionar las políticas de aprendizaje a lo largo de la vida" (párr. 3). Si bien es cierto que el cambio ha sido acelerado, y que este se ha logrado gracias a las herramientas que proporcionan las plataformas digitales, también es cierto que las desigualdades socioeconómicas y las brechas digitales entre generaciones se han hecho aún más evidentes.

En general, profesorado, familias y alumnado están sometidos a un estrés brutal. El profesorado ha tenido que reinventar en tiempo récord otra forma de impartir clase y de relacionarse con los alumnos. Lo ha hecho, en general, aportando sus medios personales y sin orientaciones claras y sin pautas de desconexión digital, sintiéndose continuamente escrutados y evaluados (Beltrán et al., 2020, p. 100).

Debido a la situación de emergencia, se ha evidenciado la existencia de algunas brechas. Fernández (31 de marzo de 2020) menciona tres:

1) La primera brecha hace referencia a la accesibilidad de internet y la posibilidad de contar con dispositivos electrónicos. El mito de la brecha insuperable se ha mantenido por inercia y porque exagerar las carencias servía a algunos docentes para justificar su inacción y a algunos alumnos para eludir tareas y exigencias.

2) La segunda brecha se relaciona con la capacidad y potencia de la red de internet, la calidad de los dispositivos con los que se cuente en casa, así como con el tiempo que cada estudiante destine a su aprendizaje dependiendo del entorno familiar

3) La tercera brecha pone de manifiesto que hay profesores y centros educativos con o sin experiencia en educación digital, con y sin una actitud innovadora, con o sin plataformas ya en marcha y probadas

Los maestros han tenido que capacitarse de manera acelerada para comenzar a ofrecer las clases que habitualmente impartían de manera presencial; se han tenido que adaptar de manera vertiginosa a las plataformas propuestas por las instituciones para evidenciar su labor educativa. La educación se ha vuelto más personal porque cada estudiante tiene diferentes particularidades, sociales, familiares y económicas. "Consideramos que los diferentes recurso que ofrece la Estrategia de Educación a Distancia: transformación e innovación para 


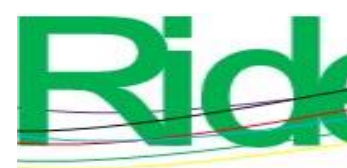

Revista Iberoamericana para la
Investigación y el Desarrollo Educativo
ISSN $2007-7467$

México, puede ser una oportunidad para recuperar la creatividad de los docentes y apuntalar su protagonismo dentro de la estrategia " (Comisión Nacional para la Mejora Continua de la Educación [Mejoredu], 2020, párr.5).

Debido a los cambios tan repentinos por los que ha tenido que atravesar la sociedad, es posible que la educación se focalice más en estimular un pensamiento crítico de la población estudiantil que permita adaptarse con más facilidad y desarrollar iniciativas para fortalecer el aprendizaje significativo, y así ser más autónomos y creativos.

Lo más importante será restablecer los paradigmas educativos: una educación menos académica, menos centrada en las disciplinas, y más práctica, más orientada hacia la comprensión del mundo que nos rodea, bajo una perspectiva de resolución de necesidades y problemas de la vida social, política, económica y del ambiente natural. La interdisciplinariedad es una herramienta muy importante para facilitar en los estudiantes una mejor comprensión. Realizar el trabajo desde casa implica romper el paradigma del trabajo in situ, por lo que las personas deben tener la capacidad de organizar sus tiempos y agenda de trabajo durante el día para evitar situaciones de ansiedad o estrés (Álvarez, 16 de abril de 2020; Ávila, 2017; Tapia, 24 de marzo de 2020).

El cierre de las escuelas en el marco de la pandemia ha implicado la creación de estrategias para continuar la enseñanza de los estudiantes en nuevas condiciones. Así los integrantes de las comunidades escolares se enfrentan a escenarios en los que cada uno asume actividades distintas a las establecidas tradicionalmente. Las maestras y los maestros afrontan una doble responsabilidad; por un lado, con su familia y seres queridos, ya que son madres o padres, cuidadores de adultos mayores, proveedores; por otro, con sus estudiantes, en tanto que encarna el desafío de coordinar y facilitar su aprendizaje escolar en el hogar y a distancia. Esto implica ensayar y perfeccionar nuevas formas de enseñanza mediante tecnologías y la necesidad de crear relaciones afectivas y pedagógicas distintas. Muchos docentes enfrentan el reto en medio de diversas limitaciones de acceso a estas tecnologías; otros, como quienes trabajan en escuelas multigrado o atienden a población migrante, afrontan un desafío aún mayor (Mejoredu, 2020, p. 8). 


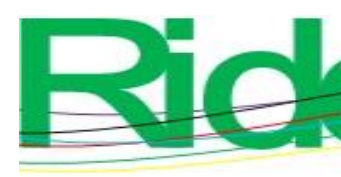
Revista Iberoamericana para la
Investigación y el Desarrollo Educativo
ISSN $2007-7467$

\section{Objetivos}

- Diagnosticar el nivel de capacitación de profesores y alumnos en el uso de las plataformas digitales propuestas por la UANL.

- Conocer y analizar las experiencias adquiridas durante la educación en tiempos de Covid-19 en la comunidad de la Facultad de Música

- Analizar las posibilidades estratégicas que se pudieron implementar durante el confinamiento sanitario

\section{Materiales y métodos}

Para este estudio de investigación-acción se utilizó una visión crítica emancipatoria sobre la base de la investigación cualitativa. "La investigación-acción crítica está íntimamente comprometida con la transformación de la organización y práctica educativa, pero también con la organización y práctica social" (Latorre, 2015, p. 31). Al respecto de la visión emancipatoria, Álvarez (citado en Hernández, Fernández y Baptista, 2010) señala que en esta el objetivo va más allá de resolver el problema o desarrollar mejoras a un proceso: pretende que los participantes generen un profundo cambio social por medio de la investigación.

En este trabajo se utilizaron tres instrumentos de medición por etapas. El primero de ellos tuvo la finalidad de hacer un primer diagnóstico acerca de las herramientas y la conectividad de la comunidad de la Facultad de Música. Posteriormente, se aplicó un segundo instrumento para observar las experiencias adquiridas durante el proceso de migración de las clases presenciales a la educación en línea. Y finalmente se aplicó un tercer instrumento para observar los resultados de la implementación de la estrategia digital propuesta por la UANL.

La investigación-acción realiza simultáneamente la expansión del conocimiento científico y la solución de un problema, mientras aumenta, igualmente, la competencia de sus respectivos participantes (sujetos coinvestigadores) al ser llevada a cabo en colaboración, en una situación concreta y usando la realimentación de la información en un proceso cíclico (Martínez, 2015, p. 239).

Para detallar más acerca de los instrumentos aquí empleados hay que decir que el primero de ellos fue un cuestionario cerrado con cinco ítems, con escala de Likert, realizado a través de un formulario de Google por parte del Departamento de Escolar de la Facultad de 


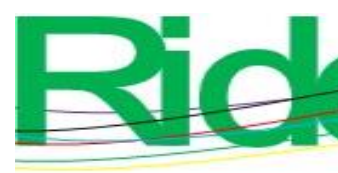

Revista Iberoamericana para la
Investigación y el Desarrollo Educativo
ISSN $2007-7467$

Música de la UANL. Se distribuyó a través de medios digitales con la finalidad de conocer el nivel de capacitación de la población académica para la utilización de la plataforma Microsoft Teams, el número de miembros que tienen internet en su hogar, las plataformas que se han utilizado y la habilitación del correo institucional. La población estudiantil es de 716 personas, la muestra de esta investigación fue de 480 participantes; el universo de profesores es de 107 personas, la muestra fue de 70 participantes. Se utilizó un análisis de resultados porcentual para realizar el diagnóstico inicial.

El segundo instrumento de medición fue un cuestionario que se aplicó con la intención de conocer y analizar las experiencias adquiridas durante el proceso de adaptación de la educación presencial a la modalidad virtual, cambio que se suscitó por la emergencia sanitaria, desde el punto de vista académico y social. Estuvo compuesto de nueve ítems; también aquí se recurrió a la escala de Likert y fue realizado a través de un formulario de Google. Los cuestionarios se enviaron en línea a través de la plataforma Microsoft Teams a 150 participantes de la Facultad de Música de la UANL. Así, se obtuvo una muestra de 132 personas, entre los que se encuentran 118 estudiantes y 14 profesores. Más adelante, se utilizó un análisis de resultados porcentual y descriptivo con la finalidad de conocer las experiencias adquiridas por los estudiantes y profesores de la Facultad de Música y analizar las posibilidades que se puedan implementar con el fin de fortalecer nuestra formación académica.

El tercer instrumento de medición fue una encuesta semiestructurada que se aplicó a 120 estudiantes de los niveles técnico medio, técnico superior y licenciatura. Constó de cinco preguntas cerradas con escala de Likert y seis preguntas abiertas. Cabe mencionar que en este tercer instrumento solamente participaron estudiantes y que se aplicó durante la semana de exámenes finales. Aquí también se realizó un análisis de resultados porcentual, uno descriptivo y un análisis del discurso para las preguntas abiertas. Como resultado se obtuvieron categorías inductivas. Lo anterior fue realizado con la finalidad de observar los resultados de la implementación de la estrategia digital propuesta por la UANL para el cierre del semestre enero-junio. "Los diseños investigación-acción también representan una forma de intervención y algunos autores los consideran diseños mixtos, pues normalmente recolectan datos cuantitativos y cualitativos, y se mueven de manera simultánea entre el esquema inductivo y deductivo" (Hernández et al., 2010, p. 514). 


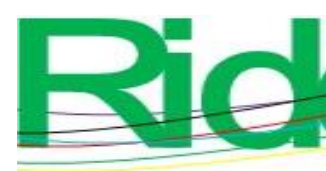

Revista Iberoamericana para la Investigación y el Desarrollo Educativo ISSN 2007 - 7467

\section{Resultados}

\section{Primer instrumento de medición}

El primer instrumento se aplicó a la comunidad estudiantil y docente con el propósito de conocer el grado de capacitación en la plataforma Microsoft Teams, propuesta por la UANL como parte de la estrategia digital para hacer frente a la contingencia por la covid-19. Esta plataforma puede ser utilizada por profesores y alumnos para dar continuidad a las actividades académicas de todos los niveles. Su acceso es a través del correo universitario, que deberá estar habilitado para cada uno de los miembros del personal académico y estudiantil.

Así pues, se hizo un diagnóstico acerca de la capacitación que tiene la comunidad académica para utilizar la plataforma Microsoft Teams, la accesibilidad de internet con la que cuentan en sus hogares, la habilitación del correo institucional y los medios por los cuales se han capacitado para utilizar esta plataforma.

El instrumento lo respondieron 480 personas (67.03\%), de un total de 716 (técnico medio, superior y licenciatura). En otras palabras, 236 alumnos no respondieron la encuesta enviada por el Departamento de Escolar de la Facultad de Música (ver tabla 1).

Tabla 1. Resumen descriptivo de la población estudiantil y porcentajes de encuestados

\begin{tabular}{|c|c|c|c|}
\hline Programa educativo & Población total & Encuestados & Porcentaje de encuestados \\
\hline Técnico medio en Música & 541 & 347 & $64.14 \%$ \\
\hline $\begin{array}{c}\text { Técnico superior } \\
\text { universitario }\end{array}$ & 22 & 21 & $95.45 \%$ \\
\hline Licenciatura & 153 & 112 & $73.20 \%$ \\
\hline Total & 716 & 480 & $67.03 \%$ \\
\hline
\end{tabular}

Fuente: Departamento de Escolar de la Facultad de Música de la UANL

La mayoría de los encuestados afirma no estar capacitado en la utilización de la plataforma Microsoft Teams que propone la UANL para la realización de las clases virtuales en todos sus niveles educativos. Cabe mencionar que para acceder a esta plataforma es necesario tener el correo institucional habilitado y podemos observar que la mayoría de los estudiantes no lo tiene vigente porque no lo utiliza. Finalmente, según los resultados de este diagnóstico, la gran mayoría de los alumnos sí tiene acceso a internet en su hogar; solo una 

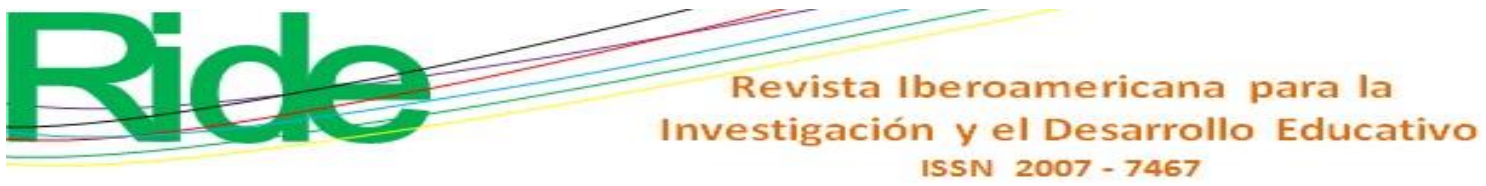

pequeña porción de personas no cuenta con una red estable para poder continuar con sus estudios de manera virtual (ver figura 1).

Figura 1. Diagnóstico de alumnos

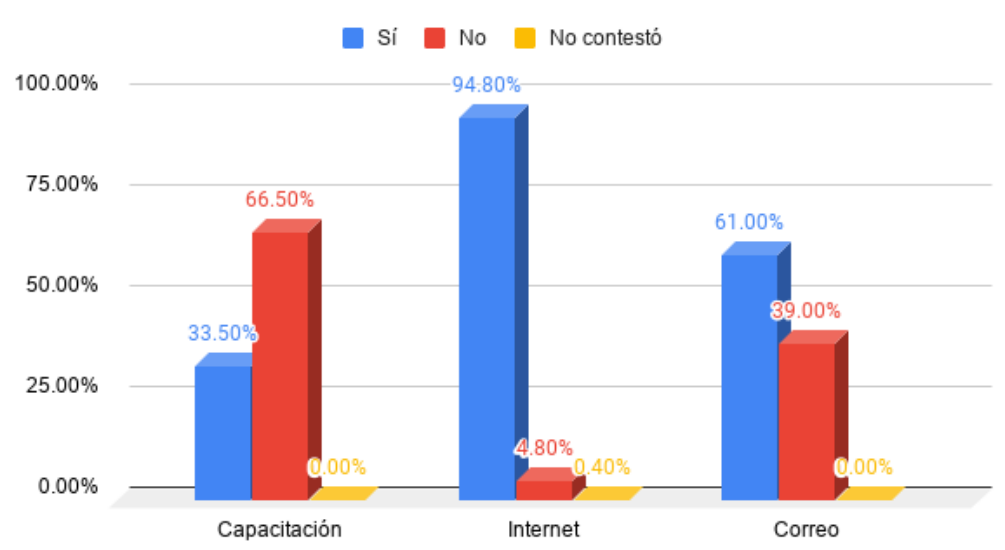

Fuente: Departamento de Escolar de la Facultad de Música de la UANL

Una vez habilitada la plataforma Microsoft Teams a través del correo institucional, el medio de capacitación más frecuentado por los alumnos ha sido directamente la utilización de esta plataforma, a pesar de que la UANL ha puesto al servicio de su comunidad académica capacitaciones programadas en línea y dentro de su plataforma de estrategia digital se han publicado manuales de libre acceso con la finalidad de facilitar el uso de esta nueva herramienta digital (ver figura 2)

Figura 2. Medios de capacitación para Microsoft Teams

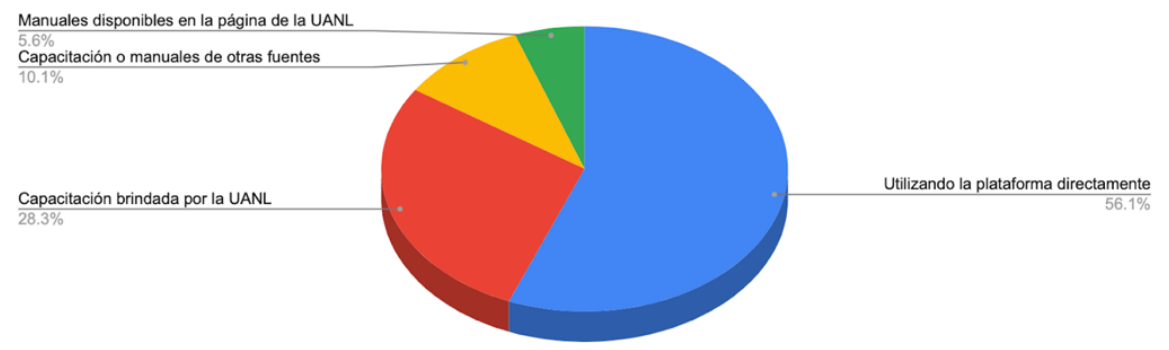

Fuente: Departamento de Escolar de la Facultad de Música de la UANL

En cuanto a los docentes, el tamaño de la muestra fue de 107 docentes de los tres diferentes niveles educativos, sin embargo, solo 70 profesores respondieron el instrumento, es decir, $65.42 \%$; el resto, 37 profesores, no contestó la encuesta (34.58 \%). 

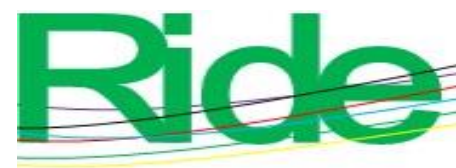

Revista Iberoamericana para la Investigación y el Desarrollo Educativo

ISSN 2007 - 7467

En la figura 3 se observa que existe un porcentaje muy similar a la muestra de los estudiantes. La mayoría de los docentes asevera no estar capacitado para el uso de esta plataforma. También se descubrió que más de la mitad de los maestros no cuenta con la habilitación del correo institucional, como en el caso de los estudiantes. Finalmente, la gran mayoría docente sí cuenta con una red de internet en su domicilio, lo que facilita la implementación de las clases en línea (ver figura 3).

Figura 3. Diagnóstico de docentes

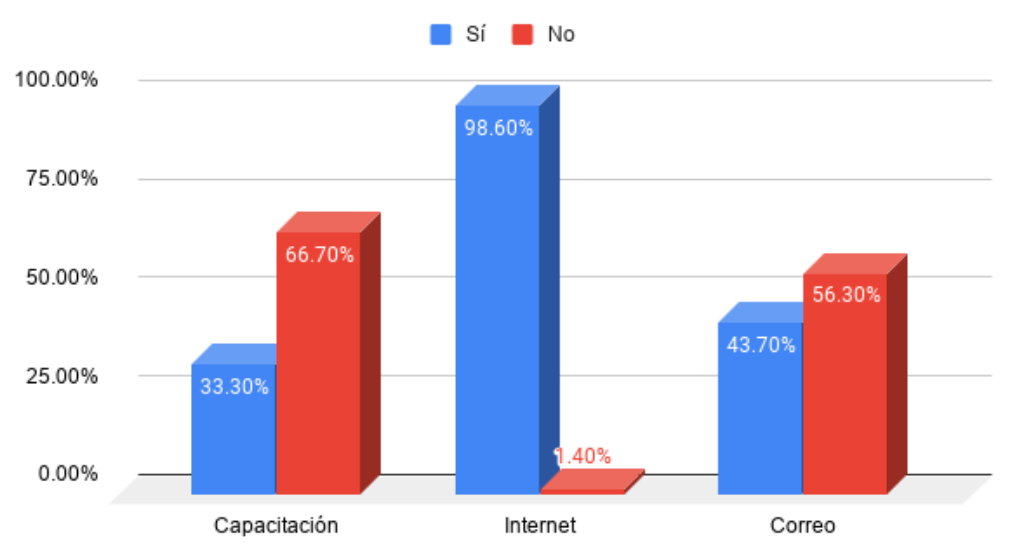

Fuente: Departamento de Escolar de la Facultad de Música de la UANL

Los medios de capacitación más frecuentados por la muestra de profesores han sido principalmente la capacitación brindada por la UANL a través de cursos y los manuales dispuestos en la página de la estrategia digital, a diferencia de los estudiantes, quienes en su mayoría se capacitaron directamente en la utilización de la plataforma Microsoft Teams (ver figura 4).

Figura 4. Medios de capacitación para Microsoft Teams

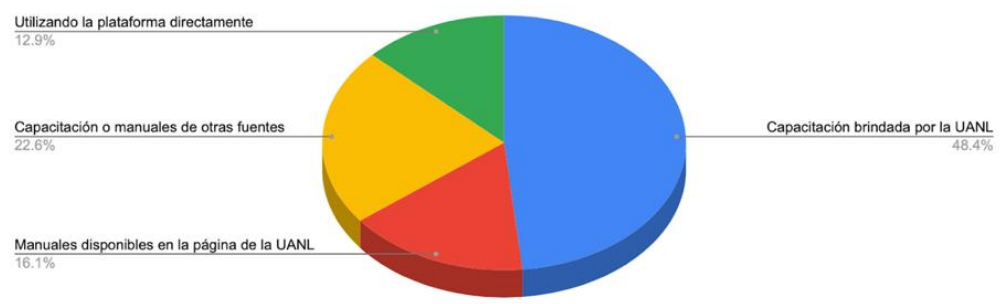

Fuente: Departamento de Escolar de la Facultad de Música de la UANL 

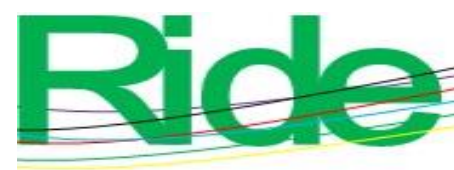

Revista Iberoamericana para la Investigación y el Desarrollo Educativo ISSN 2007 - 7467

Mientras que a partir de la segunda parte del cuestionario fue posible percatarse de que la mayoría piensa que la educación a distancia hace evidentes las diferencias socioeconómicas. Asimismo, no cree que se haya trabajado el total de los contenidos temáticos previstos pertenecientes al mapa curricular del semestre en cuestión y considera que las plataformas utilizadas para su clase de instrumento no siempre son óptimas o funcionales porque a veces falla el sonido o se desplaza (ver figura 6).

Figura 6. Diferencias económicas, contenido curricular y uso de las plataformas

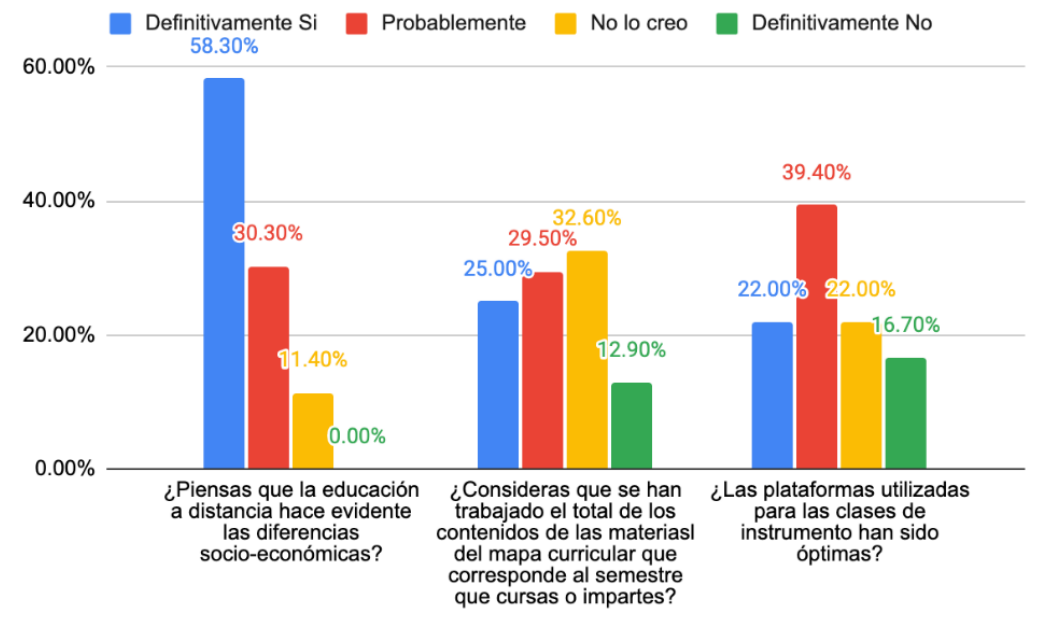

Fuente: Elaboración propia

Respecto a la última parte del cuestionario, la mayoría de los participantes considera que la educación musical debe de tener una adecuación en la utilización de las tecnologías digitales. Opina, además, que definitivamente sí es necesario que los profesores y estudiantes tengan una mayor capacitación dentro de las plataformas que se están utilizando y finalmente valora que la información de las estrategias implementadas por la Facultad de Música hayan sido claras (ver figura 7). 

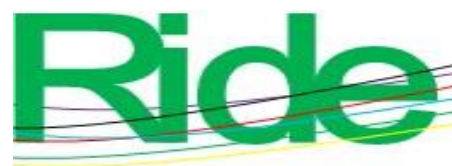

Revista Iberoamericana para la Investigación y el Desarrollo Educativo ISSN 2007 - 7467

Figura 7. Información, capacitación y utilización de las plataformas en la educación

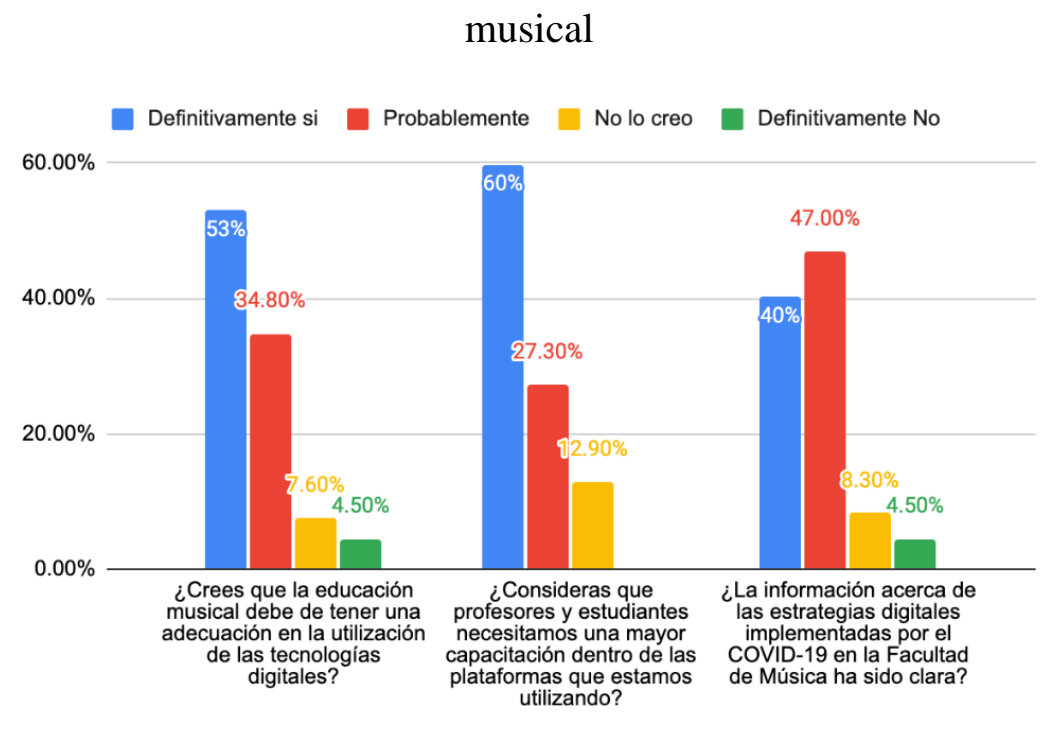

Fuente: Elaboración propia

\section{Tercer instrumento de medición}

El tercer instrumento de medición se implementó con el objetivo de analizar las posibilidades estratégicas que se implementaron a partir del confinamiento sanitario. Se realizó a través de una encuesta semiestructurada que se aplicó en línea a 120 estudiantes de los niveles técnico medio, técnico superior y licenciatura durante la semana de exámenes finales (del 1 al 5 de junio).

En la participación de la encuesta hubo $50.8 \%$ de hombres y $49.2 \%$ de mujeres. La mayoría de los encuestados pertenece a técnico medio 77\%; los niveles licenciatura y técnico superior registraron $18.6 \%$ y $3.4 \%$, respectivamente.

Como parte de los resultados, la mayoría de los estudiantes considera que ha administrado adecuadamente el tiempo para realizar sus estudios durante el confinamiento; hay un gran porcentaje de alumnos que trabajan además de estudiar, y según la opinión de los participantes, la red de internet que han utilizado para sus clases virtuales ha sido efectiva (ver figura 8). 

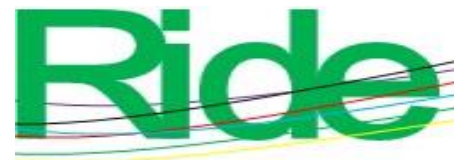

Revista Iberoamericana para la Investigación y el Desarrollo Educativo

ISSN 2007 - 7467

Figura 8. Administración del tiempo, trabajo y capacidad de internet

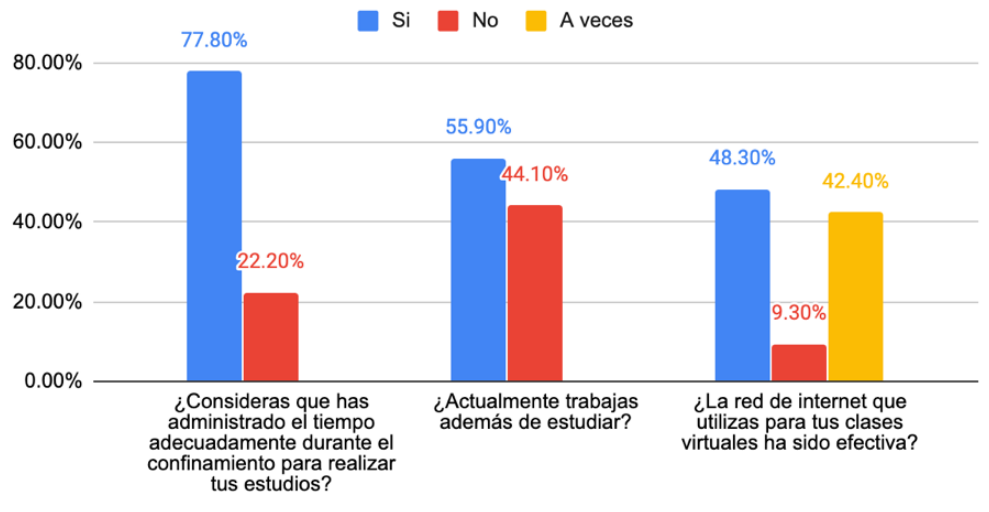

Fuente: Elaboración propia

Igualmente, para la mayoría de los estudiantes, la educación a distancia ha provocado un mayor estrés. Asimismo, los participantes, en su mayoría, demuestran una gran preferencia por llevar un sistema de educación híbrido, algunas clases presenciales y otras virtuales.

Figura 9. Estrés en los estudiantes y preferencia por un modelo híbrido

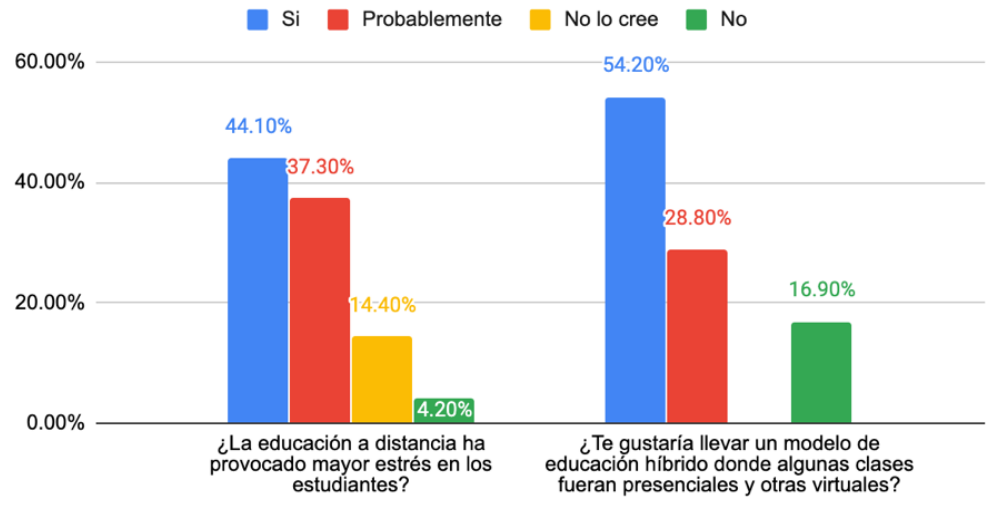

Fuente: Elaboración propia 


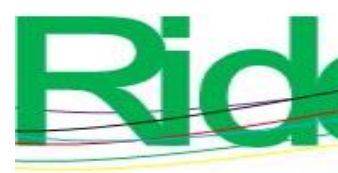

Revista Iberoamericana para la Investigación y el Desarrollo Educativo ISSN 2007 - 7467

\section{Análisis del discurso de las preguntas abiertas \\ Menciona cinco ventajas que se obtuvieron durante las clases virtuales}

Tabla 2. Cinco ventajas que se obtuvieron durante las clases virtuales según la población estudiantil

\begin{tabular}{|l|l|}
\hline Ventajas que más se mencionaron & Frecuencias \\
\hline Ahorro de tiempo & 114 \\
\hline Ahorro en transporte & 92 \\
\hline Más tiempo para practicar el instrumento & 87 \\
\hline Más contacto con los maestros & 76 \\
\hline Comer y dormir mejor & 75 \\
\hline
\end{tabular}

Fuente: Elaboración propia

Algunos estudiantes mencionaron también que las grabaciones de las clases virtuales fueron de mucha ayuda para poder avanzar mejor, que se sintieron más seguros por no tener que salir de casa, porque tuvieron menor riesgo de contagio, y que los profesores se mostraron más flexibles.

\section{Menciona cinco desventajas que observaste durante las clases virtuales}

Tabla 3. Cinco desventajas que observaste durante las clases virtuales

\begin{tabular}{|l|l|}
\hline Desventajas que más se mencionaron & Frecuencias \\
\hline Fallas en la plataforma derivadas de la mala conexión de internet & 106 \\
\hline La plataforma no funciona para clases de instrumento & 98 \\
\hline Trabajo excesivo en las materias teóricas & 62 \\
\hline Espacio inadecuado para recibir las clases & 46 \\
\hline La retroalimentación no es inmediata & 44 \\
\hline
\end{tabular}

\section{Fuente: Elaboración propia}

Sin embargo, los alumnos también manifestaron que la plataforma falla mucho a causa de la red de internet. En ocasiones se ralentiza o se pierde y en muchas ocasiones se congela la imagen o se desfasa el sonido, motivo por el cual las clases de instrumento son muy incómodas, porque no hay continuidad en el sonido, es de muy mala calidad, además de que se necesitarían varias cámaras para que el maestro pudiera observar la postura 


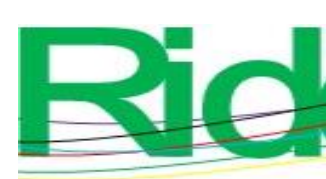

\section{Revista Iberoamericana para la Investigación y el Desarrollo Educativo ISSN 2007 - 7467}

corporal. Se ha optado por el envío de videos, aunque en este caso la retroalimentación no es inmediata.

Muchos estudiantes comentaron que su casa es pequeña, que tienen hermanos estudiando o que los padres trabajan también en casa, por lo que sienten que no tienen un espacio funcional para sus clases. Finalmente, se sintieron muy estresados porque la carga de trabajos de algunas materias consideran que fue excesiva.

\section{Comparte cinco estrategias que utilizaste para el estudio del instrumento}

Tabla 4. Comparte cinco estrategias académicas que utilizaste para el estudio del instrumento

\begin{tabular}{|l|l|}
\hline Estrategias utilizadas & Frecuencias \\
\hline Administración del tiempo & 112 \\
\hline Escuchar grabaciones de las obras & 114 \\
\hline Estudiar con metrónomo & 93 \\
\hline Consultar las grabaciones de las clases virtuales & 72 \\
\hline Tener más contacto con el maestro de instrumento & 54 \\
\hline
\end{tabular}

Fuente: Elaboración propia

La mayoría de los alumnos mencionó la importancia de la organización en el tiempo de estudio, establecer metas diarias para poder abordar su material; casi todos se auxiliaron a través de grabaciones, se acostumbraron a utilizar el metrónomo como herramienta de trabajo y mencionaron que, como la conexión no era buena durante su clase de instrumento, tuvieron que enviar videos para que el maestro viera su avance real y esperar la retroalimentación, a la cual, por el lado bueno, podían consultar en repetidas ocasiones. 


\section{¿Qué clases te gustaría tomar virtualmente?}

Tabla 6. ¿Qué clases te gustaría tomar virtualmente?

\begin{tabular}{|l|l|}
\hline Clases virtuales preferidas & Frecuencias \\
\hline Todas las materias teóricas & 37 \\
\hline Historia de la Música & 34 \\
\hline Armonía & 26 \\
\hline Solfeo & 22 \\
\hline
\end{tabular}

\section{Fuente: Elaboración propia}

Los estudiantes opinaron que las clases que prefieren tomar virtualmente son la mayoría de las materias teóricas; mencionaron primeramente Historia de la Música, Apreciación Musical, Armonía y Solfeo. Solamente 10 \% expresó que no le gustan las clases en línea y que prefiere la modalidad presencial. Las clases pudieran ser sincrónicas y asincrónicas para reforzar los contenidos de las materias teóricas.

\section{¿Qué clases preferirías tomar presencialmente?}

Tabla 7. ¿Qué clases preferirías tomar presencialmente?

\begin{tabular}{|l|l|}
\hline Clases presenciales preferidas & Frecuencias \\
\hline Instrumento & 70 \\
\hline Solfeo & 43 \\
\hline Conjuntos Corales & 42 \\
\hline Orquesta y Ensambles & 24 \\
\hline Todas las clases prácticas & 27 \\
\hline
\end{tabular}

Fuente: Elaboración propia

La mayoría de los participantes expresó que las clases de instrumento deberían de ser presenciales. En la materia Solfeo se puede observar que un gran porcentaje prefiere tener la clase de manera presencial; Conjuntos Corales también tiene un gran porcentaje de preferencia en presencial. En general, observamos que en las materias prácticas se hace necesario tener un contacto directo con los estudiantes, por lo menos en algunas frecuencias durante el semestre. 


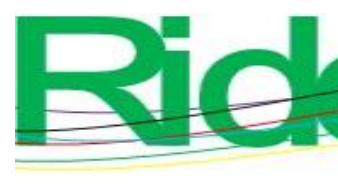

Revista Iberoamericana para la Investigación y el Desarrollo Educativo ISSN 2007-7467

\section{Discusión}

Se diseñaron y aplicaron tres instrumentos de medición en el contexto de la educación en línea en la Facultad de Música derivada de la emergencia sanitaria causada por la covid19. Gracias al primer instrumento de medición pudimos observar que la mayoría de los estudiantes y profesores tienen una red de internet estable en su domicilio; sin embargo, se detectó que la gran parte de la comunidad académica no contaba con la habilitación del correo institucional necesaria para la utilización de la plataforma Microsoft Teams y tampoco estaban capacitados para la utilización de esta plataforma propuesta por la UANL como parte de la estrategia digital. Según Rogero (2020), algunos análisis sobre la situación del alumnado durante el confinamiento han puesto el foco en la brecha digital, es decir, en el acceso a dispositivos digitales, el conocimiento sobre su uso y la disponibilidad de contenidos y metodologías adaptados al aprendizaje a distancia.

A partir de este cambio de modalidad en la educación, prevalece, además de la flexibilidad académica, la grabación de las sesiones virtuales en las que los estudiantes pueden reforzar su conocimiento, fomentar el aprendizaje mutuo, emplear estrategias de aprendizaje autónomo que les permitan el desarrollo de la creatividad y la curiosidad, mostrar autodisciplina, lo que fomentará a los estudiantes un mayor desarrollo y capacidad de distribución y organización del tiempo.

Para Koutsoupidou (2009, 2010), la tecnología puede ser valiosa para los profesores de música, ya que proporciona un banco de recursos, paquetes de software y aplicaciones. Por lo tanto, potencialmente, puede aumentar su confianza al reemplazar las formas convencionales de enseñanza que requieren ciertos niveles de conocimiento y competencias musicales

Finalmente, como asevera Dammers (2009), la interpretación musical es inherentemente una experiencia sincrónica, ya que los músicos hacen música juntos a un tiempo. Mientras muchos programas universitarios en otras disciplinas se están moviendo rápidamente hacia la instrucción asincrónica en línea, es poco probable que los programas de música lo hagan, dada su naturaleza sincrónica. La mayoría de los encuestados opina que en las clases de instrumento las plataformas digitales no son lo suficientemente sólidas para poder trabajar el sonido, la musicalidad y la posición corporal; tampoco son eficientes en clases como ensambles o coro, donde los estudiantes deben de trabajar conjuntamente para lograr un producto musical de calidad. 


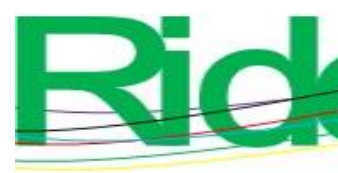

Revista Iberoamericana para la
Investigación y el Desarrollo Educativo
ISSN $2007-7467$

En el contexto del Reino Unido, los profesores defienden la modalidad virtual en materias teóricas, sin embargo, no la creen adecuada para las clases de instrumento, a menos de que sea a través de grabación de video (Palau, Usart y Ucar, 2020).

\section{Conclusiones}

A partir del primer instrumento de medición se diagnosticó que la mayoría de la comunidad docente y estudiantil de la Facultad de Música cuenta con internet en su hogar para poder acceder a la plataforma Microsoft Teams propuesta por la UANL. El principal obstáculo que se presentó fue la falta de capacitación de la plataforma debido a la premura con la que se implementó: un gran porcentaje de encuestados manifestó haberse habilitado directamente en la plataforma, no obstante se ofrecieron capacitaciones mediante videos tutoriales y asesoría virtual calendarizada a través de la estrategia digital para la contingencia covid-19. Otro factor importante que ralentizó el comienzo fue que $39 \%$ de los estudiantes y $56.30 \%$ de los profesores no tenía habilitado su correo institucional necesario para el acceso a la plataforma.

Para conocer y analizar las experiencias adquiridas durante la educación en tiempos de covid-19 en la Facultad de Música, se diseñó un segundo instrumento de medición cuyos resultados ponen de manifiesto que la mayoría de los participantes no se sienten seguros de estar preparados para la educación a distancia. Un gran porcentaje considera que el conocimiento se ha vuelto más autónomo debido a la implementación de las clases virtuales. La intervención de los profesores ha sido creativa e innovadora porque han tenido que adaptar su cátedra y cambiar las estrategias para lograr un aprendizaje significativo para el abordaje total de los contenidos de las materias del mapa curricular de cada semestre, sin embargo, algunos estudiantes piensan que no se han logrado trabajar el total de los contenidos debido al cambio de modalidad.

Es necesario tener una adecuación en la utilización de las tecnologías digitales para la educación musical, en especial para las clases de instrumento, porque las plataformas utilizadas no son óptimas, el internet falla constantemente y el sonido se atrasa. Aunado a lo anterior, de acuerdo con los resultados de los participantes, se necesita una mayor capacitación para profesores y alumnos. 


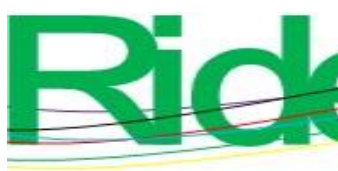

Revista Iberoamericana para la Investigación y el Desarrollo Educativo ISSN $2007-7467$

\section{Referencias}

Álvarez, G. (16 de abril de 2020). Covid-19. Cambiar de paradigma educativo. Consejo Mexicano de Investigación Educativa. Recuperado de http://www.comie.org.mx/v5/sitio/2020/04/16/covid-19-cambiar-de-paradigmaeducativo/

Ávila, B. R. (2017). Experiencias pedagógicas significativas de educación rural en Colombia, Brasil y México. Revista del Centro de Investigación de la Universidad la Salle, 12(48), 121-158.

Beltrán, J., Venegas, M., Villar, A., Andrés, S., Jareño, D. y García, P. (2020). Educar en época de confinamiento: La tarea de renovar un mundo común. Revista de Sociología de la Educación (RASE), 13(2), 92-104. Recuperado de https://doi.org/10.7203/RASE.13.2.17187.

Cárdenas, S. y Valdés, R. (15 de abril de 2020). La educación a lo largo de la vida en los próximos años. Nexos. Recuperado de https://educacion.nexos.com.mx/?p=2262.

Comisión Nacional para la Mejora Continua de la Educación [Mejoredu]. (2020). 10 Sugerencias para la educación durante la emergencia por COVID-19. México: Gobierno de México. Recuperado de https://www.gob.mx/mejoredu/articulos/10sugerencias-para-la-educacion-durante-la-emergencia-por-covid-19?idiom=es.

Dammers, R. J. (2009). Utilizing Internet-Based Videoconferencing for Instrumental Music Lessons. Update: Applications of Research in Music Education, 28(1), 17-24. Retrieved from http://doi.org.conricyt.remotexs.co/10.1177/8755123309344159.

Feito, R. (2020). Este es el fin de la escuela tal y como la conocemos. Unas reflexiones en tiempo de confinamiento. Revista de Sociología de la Educación (RASE), 13(2), 156163. Recuperado de http://dx.doi.org/10.7203/RASE.13.2.17130.

Fernández, M. (31 de marzo de 2020). Una pandemia imprevisible ha traído la brecha previsible. Cuaderno de campo. Recuperado de https://bit.ly/2VT3kzU.

Giráldez, A. (coord. ${ }^{a}$ (2010). Música. Investigación, innovación y buenas prácticas. España: Grao.

Green, L. (2019). El aprendizaje de la música pop. Avanzando en la educación musical. Madrid, España: Morata

Hernández, R., Fernández, C. y Baptista, P. (2010). Metodología de la investigación. México: McGraw-Hill. 


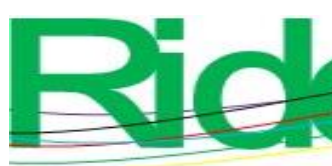

Revista Iberoamericana para la
Investigación y el Desarrollo Educativo ISSN $2007-7467$

Hernández, R. y Mendoza, C. (2018). Metodología de la investigación. Las rutas cuantitativa, cualitativa y mixta. México: Mc Graw Hill.

Koutsoupidou, T. (2009). El mundo digital de los niños: integrando música y matemáticas en educación preescolar. En Addessi, A. R. y Young, S. (eds.), Actas de la 4. ${ }^{a}$ Conferencia de la Red Europea de Educadores e Investigadores de Música de Niños Pequeños (pp. 375-384). Bolonia, Italia: Universidad de Bolonia.

Koutsoupidou, T. (2010). Formación musical inicial de maestros generalistas de jardín de infantes en Grecia: ¿Qué piden y qué reciben? Revisión de la política de educación artística.

Koutsoupidou, T. (2014). Online distance learning and music training: benefits, drawbacks and challenges. Open Learning, 29(3), 243-255. Retrieved from https://doi.org/10.1080/02680513.2015.1011112

Latorre, A. (2015). La investigación-acción. Conocer y cambiar la práctica educativa. Barcelona, España: Graó

Malbrán, S. (2007). El oído de la mente. Madrid, España: Akal.

Palau, R. F., Usart, M. y Ucar, M. J. (2019) La competencia docente digital de los docentes de los conservatorios. Estudio de autopercepción en España. Revista Electrónica de LEEME, (44), 24-41.

Martínez, M. (2015). Ciencia y arte en la metodología cualitativa. México:Trillas

Milenio Digital. (14 de marzo de 2020). Cancelan clases en la UANL para prevenir contagio de coronavirus. Milenio. Recuperado de https://www.milenio.com/ciencia-ysalud/sociedad/coronavirus-mexico-uanl-cancela-clases-aviso.

Organización Mundial de la Salud [OMS]. (2020). Brote de enfermedad por coronavirus (COVID-19). Recuperado de https://www.who.int/es/emergencies/diseases/novelcoronavirus-2019.

Rogero, J. (2020). La ficción de educar a distancia. Revista de Sociología de la Educación (RASE), 13(2), 174-182. Recuperado de http://dx.doi.org/10.7203/RASE.13.2.17126.

Secretaría de Educación Pública. (16 de marzo de 2020). ACUERDO número 02/03/20 por el que se suspenden las clases en las escuelas de educación preescolar, primaria, secundaria, normal y demás para la formación de maestros de educación básica del Sistema Educativo Nacional, así como aquellas de los tipos medio superior y superior dependientes de la Secretaría de Educación Pública. Diario Oficial de la Federación. Recuperado

de 

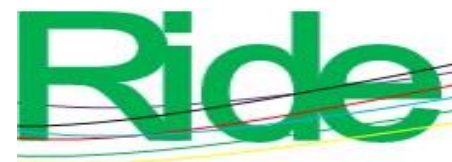

Revista Iberoamericana para la Investigación y el Desarrollo Educativo ISSN $2007-7467$

https://www.gob.mx/cms/uploads/attachment/file/543994/Acuerdos_Covid_19_DO F_27_03_20.pdf.pdf.pdf.pdf

Silva, R, y Juárez, J. (2013). Manual del modelo de documentación de la Asociación de Psicología Americana (APA) en su sexta edición. México, Puebla: Centro de Lengua y Pensamiento Crítico.

Tapia, I. (24 de marzo de 2020). Home office durante el coronavirus ¿Estás preparado? Educación Futura. Recuperado de https://www.educacionfutura.org/home-officedurante-el-coronavirus-estas-preparado/.

Torales, B. y Munguía, M. (2020). La movilidad urbana como pieza clave para la contención de pandemias. Avance y Perspectiva, 6(1). Recuperado de https://avanceyperspectiva.cinvestav.mx/la-movilidad-urbana-como-pieza-clavepara-la-contencion-de-pandemias/.

Universidad Autónoma de Nuevo León [UANL]. (2020). Estrategia digital para contingencia COVID-19. Recuperado de https://www.uanl.mx/wpcontent/uploads/2020/03/ESTRATEGIADIGITAL.pdf.

Universidad Autónoma de Nuevo León [UANL]. (2020b). Educación digital. Recuperado de http://ded.uanl.mx/estrategia-digital-uanl-2/ 


\begin{tabular}{|l|l|}
\hline Rol de Contribución & $\begin{array}{c}\text { Revista Ineroamericana para la } \\
\text { Ins el Desarrollo Educativo } 2007-7467\end{array}$ \\
\hline Conceptualización & Autor (es) \\
\hline Metodología & Beania Salcedo Moncada \\
\hline Software & Beania Salcedo Moncada 50\% José María López Prado 50\% \\
\hline Validación & José María López Prado \\
\hline Análisis Formal & Beania Salcedo Monncada 50\% José María López Prado 50\% \\
\hline Investigación & Beania Salcedo Moncada \\
\hline Recursos & Beania Salcedo Moncada \\
\hline Curación de datos & José María López Prado \\
\hline $\begin{array}{l}\text { Escritura - Preparación del } \\
\text { borrador original }\end{array}$ & José María López Prado \\
\hline $\begin{array}{l}\text { Escritura - Revisión y } \\
\text { edición }\end{array}$ & Beania Salcedo Moncada \\
\hline Visualización & José María López Prado \\
\hline Supervisión & Beania Salcedo Moncada 50\% José María López Prado 50\%. \\
\hline Administración de Proyectos & Beania Salcedo Moncada 50\% José María López Prado 50\% \\
\hline Adquisición de fondos & José María López Prado \\
\hline
\end{tabular}

